\title{
The effects of associative strength and two types of language-habit "priming" on transfer of training
}

L. R. GOULET AND JEFFREY A. GRIMM WEST VIRGINIA UNIVERSITY
Paired-associate (PA) transfer was studied under conditions where the interference effects from List 1 were pre-experimental language habits of either high or low associative strength. Subjects assigned to PA treatments received 10 trials of PA practice involving strongly or weakly associated pairs and then learned a List 2 involving identical stimuli and unrelated (number) responses. Different groups of $S s$ (Fam treatments) received 10 free recall trials on List 1 " $\mathrm{B}$ " responses and then learned the above List 2 where again the stimuli were strongly or weakly associated to the " $B$ " responses on List 1. Parallel degrees of negative transfer occurred under the PA treatments independently of List 1 associative strength. However, negative transfer varied directly with associative strength under the Fam treatments. This result provided evidence for the covert elicitation of pre-experimental language associates during free recall learning.

There has been considerable recent interest in the effects of pre-experimental language habits on verbal learning. However, the research comparing pairedassociate (PA) learning as a function of associative strength has been somewhat equivocal. Postman (1962), using adults as Ss, has found that PA learning varies directly with associative strength only when the lists were comprised of low frequency materials. There is no apparent reason why PA learning does not vary with associative strength when high frequency materials are used. One possibility is that the associative strength of language habits of high frequency materials may be sufficiently strong to allow for the learning of a PA list within one or two trials independently of the associative strengths of the pairs. This hypothesis is tenable especially since the PA learning of children (whose language habits may be assumed to be weaker than those of adults) is a direct function of associative strength (Wicklund; Palermo, \& Jenkins, 1964).

Spence (1963) and Tolchin \& Ceraso (1964) have also found that pre-experimental language habits provide strong sources of interference under the $A-B$, A-C paradigm. In fact, Ceraso demonstrated strong negative transfer on List 2 even after one trial of List 1 practice on PA pairs that were associatively related. The present experiment permitted the assessment of transfer effects under the A-B, A-C paradigm where the sources of interference (A-B) were language habits of either strong or weak associ- ative strength. This comparison was made under the two methods of List-1 practice. The first method (PA) involved conventional PA learning where Ss received 10 trials of List-1 practice on five pairs of strong and five of weak associative strengths. The second method (Fam) involved 10 familiarization (free recall) trials on the $B$ responses, after which $\mathrm{Ss}$ learned List 2 (A-C) to criterion.

Pronounced negative transfer was expected on List 2 under both the PA and Fam treatments. This was expected for the PA treatments independently of the degree of associative strength of the List-1 pairs because of the List-1 (A-B) practice. On the other hand, greater interference may be expected on the transfer task when high-strength pairs constitute the source of competition.

The analysis relating to the Fam treatments is considerably more complex. The possibility remains that the interference found in the Spence and Tolchin \& Ceraso experiments occurred only because the specific language habits (A-B) were "primed" (i.e., practiced) in the laboratory. In other words, one or more anticipation trials may bring a specific language habit considerably above threshold, especially when high frequency materials are used.

The Fam treatment does not involve overt practice of the specific A-B pairs and such priming of the language habit, $A-B$, would be expected only if the response $B$ elicited its associate, A, during the List-1 free recall trials. Furthermore, such implicit priming of language habits should be a direct function of the associative strength between the two items; thus, less negative transfer should occur on List 2 for those pairs involving low strength responses on List 1. Method

Materials. Two sets of 10 stimulus words (nouns) were selected from the Palermo-Jenkins (1964) norms such that the formal similarity between and within sets was minimized. These sets were used as stimulus words to construct four 10-pair PA lists, two from each set of stimuli. Five of the stimulus words in each list were paired with their primary associate ( $\vec{X}$ associative strength $=43.13 \%$ ), and five with an associate of low strength $(\bar{X}=0.38 \%)$. The lists were counterbalanced such that the stimulus words paired with primary (H) associates in one list were paired with low (L) strength associates in another list and vice versa. 1 
Table 1. Summary Data for List-1 and List-2 Leaming

\begin{tabular}{|c|c|c|c|c|c|c|c|}
\hline \multirow{3}{*}{$\mathrm{PA}$} & & \multicolumn{4}{|c|}{ List I } & \multicolumn{2}{|c|}{ List 2} \\
\hline & & \multicolumn{2}{|c|}{ Trials 1.9} & \multicolumn{2}{|c|}{ Trial 10} & & $1-5$ \\
\hline & & $E$ & C & E & C & $E$ & C \\
\hline & $\mathrm{H}$ & 42.87 & 43.31 & 3.94 & 4.38 & 8.25 & 15.81 \\
\hline & $L$ & 41.19 & 42.25 & 4. 19 & 4.44 & 9.00 & 16.81 \\
\hline \multirow{2}{*}{\multicolumn{8}{|c|}{ List-1 }} \\
\hline & & \multirow{2}{*}{\multicolumn{4}{|c|}{ List 1}} & \multirow{3}{*}{\multicolumn{2}{|c|}{$\begin{array}{l}\text { List } 2 \\
\text { Trials 1-5 }\end{array}$}} \\
\hline & & & & & & & \\
\hline \multirow[t]{4}{*}{ Fam } & & \multicolumn{2}{|c|}{ Trials $1-9$} & \multicolumn{2}{|c|}{ Trial 10} & & \\
\hline & & $\mathbf{E}$ & C & $E$ & C & $\mathrm{E}$ & C \\
\hline & $\mathrm{H}$ & 41.12 & 42.56 & 5.00 & 4.24 & 11.56 & 17.12 \\
\hline & $\mathrm{L}$ & 41.44 & 42.88 & 4.88 & 4.94 & 16.69 & 17.31 \\
\hline
\end{tabular}

The Ss assigned to the PA treatments learned one of the above PA lists and a List 2 involving the identical stimuli randomly paired with the numbers 0-9 (E treatments) or a List 2 involving unrelated stimulus terms and number responses from the second set of stimuli (C treatments). The Ss assigned to Fam treatments received 10 free recall trials on the responses of one of the four List-1 PA lists and then learned a List 2 which was identical to that of the PA treatments. Again treatments were classified as $\mathrm{E}$ or $C$, depending upon whether pre-experimental associative strength existed between List-2 stimuli and List-1 responses.

Procedure. Sixty-four general psychology students were randomly assigned to one of the combinations of type of List-1 practice-E or C-List set treatments. The Ss assigned to PA treatments received 10 trials of List-1 learning (including the first presentation) followed by a test involving free recall of the List-1 responses. The Ss assigned to Fam treatments received 10 study-test trials involving free response recall. All Ss learned the identical List 2 to a criterion of one perfect trial, or a minimum of 10 trials. The PA lists were presented at a 2:2 sec rate with a 4-sec intertrial interval (anticipation method). The study trials (Fam treatments) involved a 2-sec rate followed by unpaced recall. All lists were presented on a Lafayette memory drum in five random orders. Results and Discussion

List 1 learning. The learning rates of the $H$ and $L$ pairs in the PA treatments and for the analogous items in the Fam treatments were comparable on Trials 1-9 (Table 1). A comparison of the PA and Fam treatments on Trial 10, where the recall task was identical, yielded statistical significance only for the main effect of type of practice, $F=39.85$, df = $1 / 60, p<.001$. The greater recall observed under the Fam treatments was attributable to the shift in task in the PA treatments from PA learning (Trials 1-9) to free recall (Trial 10). That is, learning was essentially perfect on Trial 9 under both Fam and PA treatments. However, the Ss assigned to the PA treatments recalled an average of 1.40 fewer items when shifted to the free recall task. No such decrement was observed under the Fam treatment which involved free recall practice on all trials.

List 2 leaming. Negative transfer occurred under each $E$ treatment; i.e., learning was more rapid for the $C$ pairs (Table 1) than for relevant $E$ pairs, $F$ $=22.90$, $\mathrm{df}=1 / 60, p<.001$. Learning was also more rapid under the Fam treatment, $F=8.04$, df $=1 / 60, p<$ .01 , and for the low strength pairs, $F=14.76, \mathrm{df}=1 / 60$, $p<.001$. The results of the present experiment are most adequately summarized by calling attention to the statistically significant three-way interaction, $F=$ $7.50, \mathrm{df}=1 / 60, \mathrm{p}<.01$. This interaction was attributable to almost identical degrees of negative transfer (E-C) for the $H$ and $L$ pairs under the PA treatment and less negative transfer for the $L$ pairs than for the $H$ pairs under the Fam treatment. Comparisons of the difference between means (E-C) for each of the $\mathrm{H}$ and $L$ patrs under the PA and Fam treatments ylelded $F=10.42$, df $=1 / 120, p<.01 ; F<1$ for the $H$ and $L$ Fam treatments, respectively, and $F=19.26,20.55$, df $=1 / 120, p<.001$ for the $H$ and $L$ PA treatments.

The negative transfer observed under the Fam treatment provides evidence for the covert elicitation of pre-experimental language associates during free recall learning. This effect may be assumed to have provided implicit practice on the specific A-B pair even though only the response, $B$, was explicitly practiced. However, an alternative explanation should be considered. It is possible that the free recall practice, by merely increasing the availability of the $B$ items, permitted some degree of response competition on List 2. This hypothesis is tenable, especially since some pre-experimental A-B associative strength existed prior to List-1 practice.

The differential negative transfer for the $H$ and $L$ pairs under the Fam treatment suggests that interference on List 2 is a function of the associative strength of the language habits. This effect was not apparent under the PA treatments possibly because the PA practice resulted in bringing both the $H$ and the $\mathrm{L}$ A-B pairs considerably above threshold.

\section{References}

Palermo, D. S., \& Jenkins, J. J. Word association norms: grade school through college. Minneapolis: University of Minnesota Press, 1964.

Spence, J. T. Associative interference on paired-associate bists from extra-experimental learning. J. verbal Learn. verbal Behav., $1963,2,329-338$.

Tolchin, G., \& Ceraso, J. Selective trace arousal. J. Psychol., $1965,60,239-243$.

Wicklund, D. A., Palemo, D. S., \& Jenkins, J. J. The effects of associative strength and response hierarchy on paired-associate learning. J. verbal Learn. verbal Behav., 1964, 3, 413-420.

\section{Hore}

1. The materials used in the present experiment are available from the authors. 